\title{
A prospective cohort study of school-going children investigating reproductive and neurobehavioral health effects due to environmental pesticide exposure in the Western Cape, South Africa: study protocol
}

Shala Chetty-Mhlanga ${ }^{1,2,3}$, Wisdom Basera ${ }^{1}$, Samuel Fuhrimann ${ }^{1}$, Nicole Probst-Hensch², Steven Delport ${ }^{4}$, Mufaro Mugari ${ }^{1,5}$, Jennifer Van Wyk ${ }^{1,5}$, Martin Röösli ${ }^{2,3}$ and Mohamed Aqiel Dalvie ${ }^{1 *}$ (D)

\begin{abstract}
Background: Research on reproductive health effects on children from low-level, long-term exposure to pesticides currently used in the agricultural industry is limited and those on neurobehavioral effects have produced conflicting evidence. We aim at investigating the association between pesticide exposure on the reproductive health and neurobehavior of children in South Africa, by including potential relevant co-exposures from the use of electronic media and maternal alcohol consumption.

Methods: The design entails a prospective cohort study with a follow-up duration of 2 years starting in 2017, including 1000 school going children between the ages of 9 to 16 years old. Children are enrolled with equal distribution in sex and residence on farms and non-farms in three different agricultural areas (mainly apple, table grapes and wheat farming systems) in the Western Cape, South Africa. The neurobehavior primary health outcome of cognitive functioning was measured through the iPad-based CAmbridge Neuropsychological Test Automated Battery (CANTAB) including domains for attention, memory, and processing speed. The reproductive health outcomes include testicular size in boys and breast size in girls assessed in a physical examination, and blood samples to detect hormone levels and anthropometric measurements. Information on pesticide exposure, co-exposures and relevant confounders are obtained through structured questionnaire interviews with the children and their guardians. Environmental occurrence of pesticides will be determined while using a structured interview with farm owners and review of spraying records and collection of passive water and air samples in all three areas. Pesticide metabolites will be analysed in urine and hair samples collected from the study subjects every 4 months starting at baseline.

Discussion: The inclusion of three different agricultural areas will yield a wide range of pesticide exposure situations. The prospective longitudinal design is a further strength of this study to evaluate the reproductive and neurobehavioural effects of different pesticides on children. This research will inform relevant policies and regulatory bodies to improve the health, safety and learning environments for children and families in agricultural settings.
\end{abstract}

Keywords: Pesticides, Low exposures, Reproductive health, Neurobehaviour, Co-exposures, School-going children, Rural communities, Endocrine disruption, Air and water

\footnotetext{
* Correspondence: aqiel.dalvie@uct.ac.za

${ }^{1}$ Centre for Environment and Occupational Health Research, School of Public

Health and Family Medicine, Faculty of Health Sciences, University of Cape

Town, Anzio Road, Cape Town, South Africa

Full list of author information is available at the end of the article
}

(c) The Author(s). 2018 Open Access This article is distributed under the terms of the Creative Commons Attribution 4.0 International License (http://creativecommons.org/licenses/by/4.0/), which permits unrestricted use, distribution, and reproduction in any medium, provided you give appropriate credit to the original author(s) and the source, provide a link to the Creative Commons license, and indicate if changes were made. The Creative Commons Public Domain Dedication waiver (http://creativecommons.org/publicdomain/zero/1.0/) applies to the data made available in this article, unless otherwise stated. 


\section{Background}

Chronic health effects resulting from agricultural pesticide exposure, especially at an early stage of development is an important public health concern globally $[1,2]$. Neurotoxic effects leading to learning and developmental disabilities as well as male and female reproductive and developmental adverse effects, are of particular concern with respect to exposure to pesticides for example in the chemical group of the organophosphates, carbamates and pyrethroids [3-6]. Several of these pesticides are hormonally active and listed as Endocrine Disrupting Chemicals (EDC's), which alter biological and developmental functioning at low levels $[6,7]$. These harmful pesticides may be absorbed into the organ tissues via ingestion of drinking water, food, inhalation of spray drift or dust and via absorption through skin [7-9].

South Africa, an upper middle-income country has the highest application rates of pesticides in Sub-Saharan Africa with over 3000 different types of pesticide product formulations registered, including the possible neurotoxic and EDC's active ingredients bifenthrin, chlorpyrifos, cypermethrin and mancozeb $[10,11]$. The Western Cape is an important crop farming sector in South Africa. Most of the pesticides including herbicides and insecticides are applied during the summer season [4]. Amongst the number of pesticides detected in the Western Cape area's surface and ground water that includes drinking water, are chlorpyrifos, deltermethrin, and endosulfan. Endosulfan was most often detected with levels exceeding the World Health Organisation (WHO) standards of $0.1 \mu / \mathrm{L}$ for health and safety $[12,13]$. High levels of endosulfan metabolites have also been detected in farm workers and residents of the rural Western Cape [13-15].

A cross-sectional study in the rural Western Cape on the reproductive health and development of school boys found different levels of reproductive hormones, lower sexual maturity ratings and anthropometric measurements, in boys who lived on farms compared to those who did not [16]. A case-control study in the Eastern Cape of South Africa found significant associations of birth abnormalities in the offspring of women exposed to agricultural chemicals during pregnancy, including various organophosphates, blue death (a mixture of carbaryl, carbufuran and campechlor/ toxaphene- banned in South Africa since 1970) and other insecticides [17]. Other than the case control study and the cross-sectional studies aforementioned there are no other studies amongst children in South Africa assessing the causal link between pesticide use and health outcomes. Data is especially limited on longitudinal studies amongst low to average exposure to pesticides and its effects on these health outcomes.
To understand the health effects of pesticides requires a better understanding of other factors affecting the physical and neurobehavioral development. Various studies observed associations between electronic media (e-media) use and behavioral patterns including inattention and wellbeing, mostly attributed to use of mobile phones than to radiation exposure [18-21]. Mobile phones are now as common in South Africa as is in America, and mobile phone usage in daily life is common, especially among adolescents. South Africa has 150 mobile phone use subscribers per 100 people, compared to 91 in developing countries, and 71 in Sub-Saharan Africa [22].

Furthermore, South Africa's Western Cape Province, has the highest rate of Fetal Alcohol Syndrome Disorder (FASD) in the world, with rates higher than 46 cases per 1000 births in recent studies [23]. Previous studies have shown that 46 to $51 \%$ of rural woman drink during pregnancy [23]. There are several challenges associated to these high rates particularly relevant in the context of the study areas in the Western Cape: the drinking situation was declared a public health challenge as the fight against alcohol abuse dates back to colonialism in the history of South Africans; no labor laws existed during the apartheid regime, and wages for farm laborers was remunerated in alcohol rather than money, referred to as the Dop (Afrikaans translation for drink) System [24]; FASD is associated 45 times higher amongst woman with lower socio-economic status (SES) than those in middle and upper SES [23]; and the agricultural sector in South Africa is the largest single employment sector, especially for women [25].

The primary aim of this prospective cohort study is to determine the association of agricultural pesticide exposure with reproductive development and neurobehavior of children in the rural Western Cape, South Africa, independent of co-exposures from use of e-media and maternal alcohol consumption. The secondary aim is to investigate associations of these co-exposures on reproductive, neurobehavioral development and well-being of children.

\section{Methods/design \\ Study design}

This research study has a longitudinal design comprising a baseline and a follow-up examination of a cohort of 1000 school-going boys and girls from the rural Western Cape of South Africa. The study design is illustrated in Fig. 1 and data collection tools described in detail under the "data collection tools" section below. Study participants are examined at baseline in 2017 and at follow-up in 2019 using the same exposure survey and pesticide biomonitoring exposure measures, as well as the same health outcome measure tools including the CANTAB, 


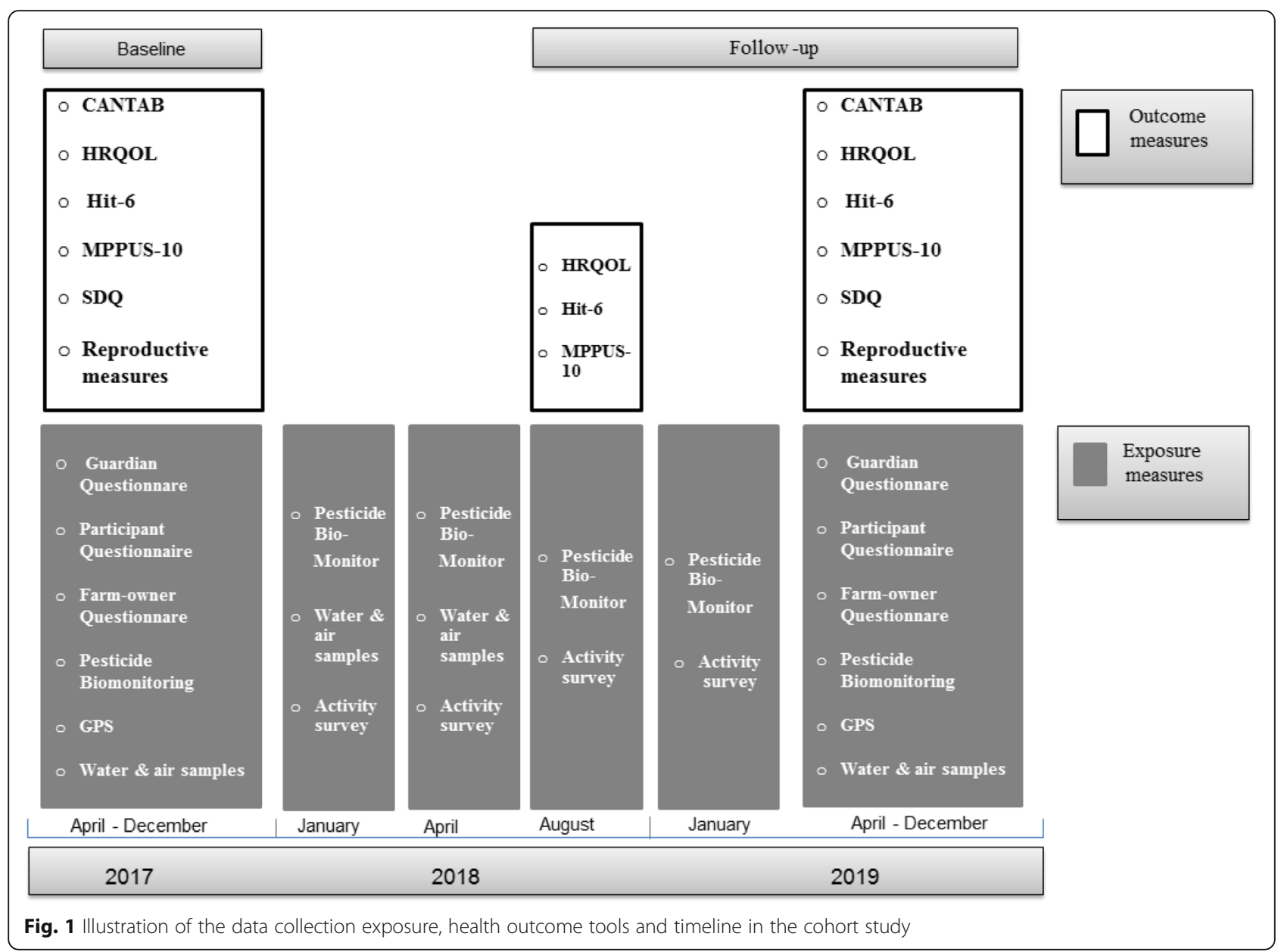

reproductive measures including i) Tanner staging - a physical examination of the male and female reproductive system (measuring sexual maturity) ii) reproductive hormone levels and iii) anthopometric measurements, Health Related Quality of Life (HRQOL, also referred to as KIDSCREEN), Headache Impact Test (HIT-6) and Problematic Mobile Phone use (MPPUS-10).

This data collection will take place in a suitable setting during school visits. The participants parent or gaurdian are also visited, to conduct an exposure survey with one health outcome tool about their child, the Strengths and Difficulties Questionnaire (SDQ). Additionally, water and air samples will be collected for 1 year (July 2017June 2018) and Global Positioning System (GPS) coordinates will be taken both at baseline and at follow-up. The farmers of the surrounding farms to the schools and those farms on which the children live, are contacted to conduct a farm survey on pesticide usage (November 2017-June 2019).

There will be four-monthly follow-ups in 2018 of pesticide exposure measures including biomonitoring (urine samples), and an exposure acitivity survey for the participants only. There will be one follow-up during
2018 for participants on three health outcome tools, the KIDSCREEN, HIT-6 and MPPUS-10.

\section{Study area}

The study is conducted in three agricultural areas: the Hex River Valley (table grapes), Grabouw (apple and stone fruits) and Piketberg (wheat and fruit) Fig. 2. The three study areas were selected according to: (i) its intensive agricultural activities applying large amount of pesticides; (ii) pesticides previously detected in the environment; and (iii) high levels of pesticide metabolites measured in workers and residents from these areas [12].

\section{Sampling}

We recruited 1000 children aged 9 to 16 years from schools in the three study areas. The children were enrolled with equal distribution per area, per gender, as well as an equal number of those living on farms and not living on farms. Of the 32 existing schools in all three study areas, only primary, intermediate and combined schools were contacted to prevent loss to follow-up (i.e., children from high schools would have left school before the follow-up examination in 2019). 


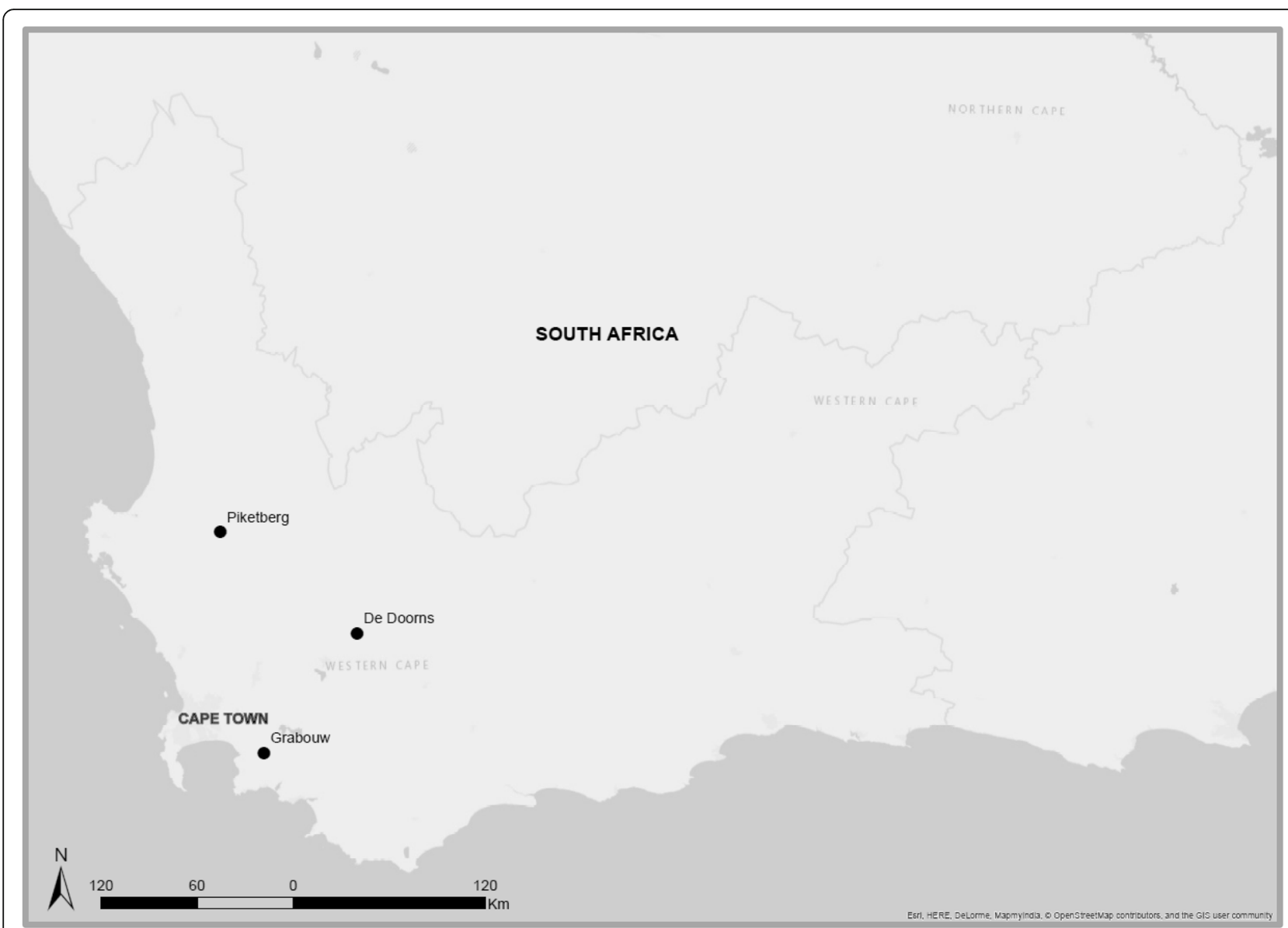

Fig. 2 Map of the three study areas, Piketberg, DeDoorns and Grabouw, in the WC of SAMap made by SM using ArcMap 10.5, @ OpenStreet Map Contributors and GIS Community

Of the 22 intermediate schools, 12 were willing to participate and four of the seven combined schools were willing to participate.

The principals and governing bodies of these 16 schools were contacted and informed about the study and seven agreed to participate. In these seven schools, information sheets about the study and the role of the school in this study together with permission letters was then sent to the parents or guardians of all the school children in grades four to nine via their children. The letter served as an invite to participate in the study which had to be indicated by signing and providing detailed contact information. In schools where the number of consenting parents or guardians exceeded the number of children targeted, random systematic sampling was to be used to select the children. Consent from parents or guardians who responded to the study invitation were obtained through home visits.

The grades were expanded from only including 4, 5 and 6 to include grades 2, 3, 7, 8 and 9. Learners from these selected schools were found in these lower and higher grades while still fitting the age criteria, 9-16 years, for the study.

\section{Data collection tools}

Neurobehavioral health outcomes measurements Cognitive assessment

The online CANTAB Connect Research, developed by Cambridge Cognition, comprise several domains of cognitive testing [26]. The CANTAB is specifically sensitive to changes in neuropscyhological performance and has been applied in over 1750 peer reviewed publications of both human and animal studies [27-29]. Cognitive domains including processing speed, attention, and memory were selected for testing in relation to targeted areas of pesticide and alcohol neurotoxicty. Two tests per domain are selected to measure specific cognitive functions within each of the three domains as shown in Table 1 [30-32]. The CANTAB has different levels for each test to accommodate age variation. The lower levels in each test were selected for this studies age range. Each test is presented to study participants through an iPad (Apple Air, 9.7") with the installed CANTAB software during the school visit. The time to complete the whole battery requires $40 \mathrm{~min}$ from each participant. Each CANTAB test measures cognitive functionining by recording 
Table 1 Cognitive domains and tests performed within the CANTAB cognitive assessment battery

\begin{tabular}{|c|c|c|c|c|c|}
\hline & Cognitive domain & Test & Cognitive function & Outcome & $\begin{array}{l}\text { Duration } \\
\text { of test }\end{array}$ \\
\hline \multirow[t]{2}{*}{1} & $\begin{array}{l}\text { Processing speed including } \\
\text { visual motor integration }\end{array}$ & Reaction Time (RTI) & $\begin{array}{l}\text { Perception of visual stimuli, } \\
\text { response to visual stimuli and } \\
\text { execution of motor action }\end{array}$ & $\begin{array}{l}\text { movement time, reaction } \\
\text { time and response accuracy; }\end{array}$ & $6 \mathrm{~min}$ \\
\hline & & Motor Screening (MOT) & $\begin{array}{l}\text { Sensorimotor or perceptual } \\
\text { motor speed and comprehension } \\
\text { difficulties }\end{array}$ & $\begin{array}{l}\text { Time lapse between display to } \\
\text { response; number of correct } \\
\text { and incorrect responses }\end{array}$ & $2 \min$ \\
\hline \multirow[t]{2}{*}{2} & $\begin{array}{l}\text { Memory including } \\
\text { executive Functioning }\end{array}$ & Spatial Working Memory (SWM)- & $\begin{array}{l}\text { Manipulation of visuo-spatial } \\
\text { information, executive demands } \\
\text { of strategy (reasoning, decision } \\
\text { making and behaviour), parts } \\
\text { of short-term memory (holding) } \\
\text { concerned with immediate } \\
\text { conscious perceptual and linguistic } \\
\text { processing }\end{array}$ & $\begin{array}{l}\text { Visits, re-visits and searches } \\
\text { for boxes }\end{array}$ & $5 \mathrm{~min}$ \\
\hline & & Paired Associate Learning (PAL) & $\begin{array}{l}\text { Visual memory and new learning, } \\
\text { episodic memory (collection of past, } \\
\text { personal experience that occurred } \\
\text { at a particular time and place with } \\
\text { associated emotions) }\end{array}$ & $\begin{array}{l}\text { Incorrect selection, adjustment, } \\
\text { problem solving and memory } \\
\text { of selection }\end{array}$ & $8 \mathrm{~min}$ \\
\hline \multirow[t]{2}{*}{3} & Attention & Attention Switching Task (AST) & $\begin{array}{l}\text { Attentional set-shifting, cognitive } \\
\text { flexibility and lateralization }\end{array}$ & $\begin{array}{l}\text { Congruency and latency } \\
\text { during change of instructions }\end{array}$ & $8 \mathrm{~min}$ \\
\hline & & $\begin{array}{l}\text { Rapid Visual Information } \\
\text { Processing (RVP) }\end{array}$ & $\begin{array}{l}\text { Sustained attention and continuous } \\
\text { performance, impulse control or } \\
\text { inhibition }\end{array}$ & $\begin{array}{l}\text { Sensitivity to target and } \\
\text { correct responses }\end{array}$ & $7 \mathrm{~min}$ \\
\hline
\end{tabular}

several outcome performance scores on each task including latency and accuracy.

\section{Health related quality of life (HRQOL)}

The comprehensive KIDSCREEN tool that measures HRQOL and wellbeing in the areas of physical, psychological, relational support and school enviroment is administered to the study participants [33]. This tool has three different versions and for the purpose of this study's time constraints, the brief general ten question tool using a five point likert scale for response, requires five minutes to complete.

\section{Problematic mobile phone use (MPPUS-10)}

The MPPUS-10 is a tool to measure problematic aspects of mobile phone use related to addiction ('withdrawel', 'loss of control', 'negative life consequences' and 'craving') and related to social components (peer dependence) [34]. A recent study on cell-phone exposure in adolsecents observed associations of MPPUS-10 with impaired psychological well-being, impaired parent and school relationships and more behavioural problems [18]. Study participants need about 5 minutes to fill in the ten items.

\section{Headache impact test (Hit-6)}

Headaches have been reported to be associated with cell-phone usage [35-37]. To address this question the HIT-6, a brief and validated tool to assess for the severity of headaches, will be applied. This six question tool, uses a five point likert scale which will take 5 minutes to complete.

\section{Strengths and difficulties questionnaire (SDQ)}

A brief version of the SDQ is administered to the guardian of the child to screen for any behavioral and affective problems in the child [38]. This widely used tool consists of five scales assessing emotional symptoms, conduct problems, hyperactivity, peer problems and prosocial behaviour on five items each answered on a three point Likert scale.

\section{Reproductive health outcome measurements Blood reproductive hormones}

During the baseline and follow-up examinations, a qualified nurse will collect early morning (before 9 am) whole blood samples $(5 \mathrm{ml})$ from 500 male participants. A sample size of 500 boys (based on findings from previous cross-sectional study [16]) is sufficient to assess differences between farm and non-farm boys. Girl participants were not sampled because of limited funding and the reality that they were less likely to undergo phlebotomy than boys. All the blood samples collected from the study site were transported to the National Health Laboratory Services (NHLS) laboratory based at the Groote Schuur Hospital in Cape Town within $24 \mathrm{~h}$ for analysis. Blood samples will be analysed for baseline follicle stimulating hormone (FSH), luteinizing hormone (LH), testosterone, estradiol (E2) and sex-hormone 
binding globulin (SHBG). Baseline hormones will be compared to age-related laboratory norm using the same assays. LH, FSH, E2 and testosterone are markers of reproductive function in boys as they are part of the Hypothalamic Pituitary Gonadal Axis and their secretion can be altered by hormonally active pesticides [5]. Significant alterations in the levels of these hormones may signal possibilities of estrogenic, anti-androgenic or other in vivo endocrine disrupting effect [6]. SHBG is measured to correct for testosterone. The NHLS laboratory does not currently have the capacity to measure inhibin, the other important male reproductive hormone that suppresses FSH for homeostatic control.

\section{Physical examination (reproductive assessment and anthropometry)}

Trained and qualified male and female nurses will physically examine the boys and girls respectively in a private room at baseline and follow-up and record the information onto a structured data collection form. Height, weight, secondary sexual characteristics and sexual maturity rating (SMR) will also be recorded [39]. The SMR will be derived by assessing penis development (testicular volume) in boys and breast development (breast size) in girls (129). Testicular volume in boys will be assessed using a standardised set of wooden testicular beads called an orchidometer [40].

Genital anatomical abnormalities including the presence of congenital hydrocoeles, undescended testes, congenital inguinal hernias and hypospadias, and the presence of infection and previous injury will also be assessed for in boys. Additionally, testicular consistency will be recorded for boys. For girls, age at menarche, length and frequency of mensuration and breast development or anatomical abnormalities will also be recorded.

Height and weight will be recorded according to standardised methods and using calibrated instruments.

\section{Assessment of self-reported exposures and other relevant information \\ Participant questionnaires}

The questionnaire administered to participant learners at baseline and follow-up include the following exposure sections:

i. Pesticide Exposure: currently living on a farm or not, recent pesticide contact include seeing and smelling pesticides, swimming in nearby dams or rivers and eating crops from the vine

ii. Farming Activities: involvement with farming activities like picking fruit, spraying, cleaning or burning containers
Confounding variables to the health outcomes in this participant questionnaire include:

iii. Injury and Other Lifestyle Activities: head injuries, sleeping difficulties and substance use.

iv. Electronic Media Use: GERoNiMO: Generalised EMF Research using Novel MethOds (including cellphones). To ascertain exposure amongst adolescents in these communities, the European Union project questionnaire, GERONIMO will be administered to enquire about whether they do use electronic media, the type of media usage, activities that they engage with on electronic media devices and the specific time spent engaged with these activities. This tool will be administered before using the MPPUS-10 described under outcome measurements and only those who indicate that they use a mobile phone will complete the MPPUS-10.

The brief pesticide exposure activity questionnaire administered to participants during the four monthly follow-ups include sections (i) and (ii) from the participant questionnaire.

\section{Guardian questionnaires}

The questionnaire administered to parent or guardian includes the following exposure sections:

i. Pesticide and Household Exposure: previous and current work and residential location, history and current exposure to pesticides during pregnancy, household chemical exposure and childhood pesticide poisoning

ii. Child Residential History: pesticide exposure in both their current and previous residence

Confounding variables to the health outcomes in this participant questionnaire include:

i. Childhood Development: including birth complications and developmental milestones of the child

ii. Medical History: including hospitalisation, diagnoses and medication

iii. Child's Diet and Nutrition

iv. Maternal Smoking and Alcohol Consumption

\section{Farm-owner questionnaire}

The farm-owner questionnaire aims to characterise farm activities in three study areas (apple, table grapes and wheat production systems). Therefore, a sub-sample of 20 farms will be selected in each area from the list of farms where children participating in the cohort study 
are living. Farm owners will be contacted via phone and a meeting will be arranged. If they are willing to participate they will be required to sign an informant consent form to agree to be part of the study to ensure confidentiality of personal data. The data on pesticide use per crop will then be used to develop spatio-temporal crop-exposure matrices (CEMs). The model will provide information on the exposure to individual agricultural pesticides according to the distance to agricultural fields and the season of the year [41].

\section{Pesticide biomonitoring}

Spot sampling of urine will be conducted per study participant by professional nurses at each sampling time point. There are five sampling time points which are, the baseline in the first year, three times in the second year and during follow-up in the third year of this project to determine short-term pesticide exposure. Approximately $8 \mathrm{ml}$ if urine will be collected from each participant. Each participant's urine sample will be separated immediately after collection at the collection point into four $2 \mathrm{ml}$ cryovials with color coded caps and stored on dry ice in a cooler box for delivery to the Hair and Skin Laboratory located at Groote Schuur Hospital, University of Cape Town. A cold chain of 2 to $8{ }^{\circ} \mathrm{C}$ will be maintained from the moment of urine seperation into cryovials at collection point within $24 \mathrm{~h}$ until storage at $-20{ }^{\circ} \mathrm{C}$ at the laboratory until they are analysed.

A hair sample, at least $200 \mathrm{mg}$ of hair, will be collected from each participant at each time point in the study, time points stated above, for determining long-term exposure to pesticides. The hair sample will be stored in a aluminium foil at room temperature until they are analysed. This sample will also be analysed by the Hair and Skin laboratory at the University of Cape Town.

The Hair and Skin laboratory will extract and analyse parent pesticides and their metabolites in the urine and hair samples. Currently analysis will be done on commonly used pesticides in the study areas. The initial pesticide analyses will focus on organophosphate metabolites, dialkyl phosphates (DAPs) and pyrethroids (screen) followed by other commonly used pesticides and more specific analyses for individual active ingredients.

In-house validated methods for extraction and analysis of DAPs in urine and hair will be used for each participant. Briefly, DAPs extraction and analysis in urine involves thawing urine samples, one color coded $2 \mathrm{ml}$ vial for each participant in a water bath. A $1 \mathrm{ml}$ volume of the urine sample will be aliquoted into a glass test tube and mixed with a DAP internal standard mixture. The mixture is freeze dried overnight, resuspended in acetonitrile and mixed by vortexing and sonication. The mixture is centrifuged for $10 \mathrm{~min}$ at $4000 \mathrm{~g}$ and the the supernantant transfered into a glass test tube and dried with a vacuum concentrator. The dried mixture is reconstituted in $200 \mu \mathrm{l}$ of mobile phase and $10 \mu \mathrm{l}$ analysed using liquid chromatography-tandem mass spectrometry (LCMS/MS).

DAPs extraction and analysis in hair involved weighing $100 \mathrm{mg}$ of hair into a Omni tube, washing the hair wit $1 \mathrm{ml}$ water followed by pulversing the hair in $1 \mathrm{ml}$ of water using an Omni BeadRuptor. The pulverised mixture is centrifuged and the supernatant is collected, filtered and subjected to LCMS/MS analysis. Methods for analysis of other pesticides and their metaboolites are currently being developed.

\section{Assessment of yearly variation of pesticides in air and water}

We will assess spatial and seasonal variations of pesticide levels in the atmosphere (over six two-month sampling rounds) and in the aquatic environment (12 one-month sampling rounds) from July 2017 to June 2018. Passive air sampling will be conducted using a total of 36 polyurethane foam disks (PUF-PAS) to sample current used pesticides and 12 PUF-PAS to sample organochlorine pesticides. Samplers will be deployed at two locations in each of the three study areas (one within $50 \mathrm{~m}$ to agricultural fields and one $>100 \mathrm{~m}$ away in a more urban village environment). Of note, PUF-PAS to measure organochlorine pesticides are only deployed at the location within $50 \mathrm{~m}$ to agricultural fields [42]. Given that there are uncertainties regarding the efficiency of sorption to PUFs of polar compounds such as pesticides, three XAD-PAS discs will be additionally deployed at sampling location within $50 \mathrm{~m}$ to the farm for 6 months to validate the PUF-PAS sampling systems [42].

Immediately after collection, the PUF-PAS or XAD-PAS samples will be put into a cool box $\left(\max 8{ }^{\circ} \mathrm{C}\right)$ and transported to the Chemical Engineering Laboratory at UCT where they will be stored at $-20{ }^{\circ} \mathrm{C}$. In addition, one blank will be distributed in each round for both current used pesticides and organochlorine pesticides. PUF disk samples including field blanks will be transported to the Research Centre for Toxic Compounds in the Environment (RECETOX) in the Czech Republic for analyses. We will target $30+$ currently used pesticides (registered and banned in South Africa) in addition to selected organochlorine pesticides which are banned for use in agriculture but may persist in the environment.

Passive water sampling will be conducted at one point downstream of the farming area within the Krom River, Hex River and Berg River located in Grabouw, the Hex River Valley, and Piketberg, respectively. Styrenedivinylbenzene (SDB) disks will be used which allows for continuous time-averaged water sampling at a monthly interval (sampling will be for 2 weeks each month) [43]. 
After 2 weeks, the SDB disks will be collected and transported to the Chemical Engineering Laboratory at UCT where they will be stored at $-20{ }^{\circ} \mathrm{C}$. In addition, one duplicate will be distributed in each round for quality control. SDB disk samples including laboratory blanks will be eventually transported to the Swiss Federal Institute of Aquatic Science and Technology (EAWAG) in Switzerland. We will target current used pesticides.

To assess water quality, we will measure water temperature, water level, conductivity and $\mathrm{pH}$ when samples are deployed and collected every second week. In addition, monitoring data of daily precipitation and water flow for the three river catchment areas will be accessed from the Department of Water and Sanitation of the Republic of South Africa (DWA).

\section{Geographical location (GPS)}

GPS coordinates of the participating children's homes will be collected during the home visits when conducting the guardian questionnaire during the baseline study. This data will be used to calculate proximity to agricultural activities (agricultural land use data are obtained via the Cape Farm Mapper which is a product of the Western Cape Department of Agriculture) which will form part of a pesticide exposure index for each participant. The GPS based proximity index will be more accurate than the one used in a previous study which was based on self reported and tape measurement information [44]. The proximity index, spraying intesity index as determined from spraying records, levels of pesticides in the environmental samples and pesticide bio-monitoring will be used to calculate a pesticide environmetal exposure index for each participant.

\section{Procedure}

All personal interviews using a structured questionnaire were installed on mobile devices using Open Data Kit (ODK) application. The GPS coordinates will also be recorded using the ODK application on the mobile phone. To ensure quality of data collection, standard operating procedures (SOPs) are developed and all field workers are trained over a week prior to data collection which will continue at different points throughout the project. The researchers were trained on the CANTAB by the Cambridge Cognition Company product specialists. Two fieldworkers were hired to conduct the CANTAB with specific criteria for the role and were trained on the CANTAB by the research team. The researcher offered continued support to the fieldworkers during the CAN$\mathrm{TAB}$ data collection, alongside the guidance of the product specialists. Fieldworkers were hired to conduct the interviews with participants, guardians and farm workers and were trained through workshops and role plays on how to conduct the interview using the ODK software with mobile phones and how to record the GPS coordinates. The study nurses to perform the physical examination were trained by an Adolescent/Child health specialist who demonstrated how to perform the anthropometric measurements, use of the orchidometer and assessment of sexual maturation using visual material. The nurses were further trained by the study co-coordinators on their role and tasks within the study as well as on the content of the tools to administer. After sufficient training for the fieldworkers on both the tools and the study itself, two pilot studies were conducted. The first pilot study to test the content and flow of the questionnaires were with 10 participants (five boys and five girls). The second pilot study was conducted on the first 100 participants to test all measurements and its work flow.

Arrangements are made with each school administration for the best logistics including days, time and place to conduct the study. The work flow on days of testing entails five separate data collection stations within appropriate private and quiet venues in each school. At the first data collection station the participants are informed for the second time about the study and given enough detail on the procedure of data collection. Written assent is required at this station. At the second station, learners are examined by a male/female nurse and have their anthropometric measurements taken. After the examination a spot urine sample is collected from boys and processed for transportation to the testing laboratories. Thereafter, they proceed to the third data collection station where learners complete the CANTAB assessment. The fourth station requires completion of the survey with a fieldworker. The fifth station entails a hair and blood sample by a nurse with the final station creating a space for the learner to debrief if needed and receive a treat for their contribution. All exposure and outcome measurements will require an hour and half from each participant and the study aims to reach 25 participants in 1 day.

Following the first phase of data collection from learners at the school, the second phase includes home visits by the fieldworkers. Here the parent/gaurdian will be interviewed which requires an hour to complete the questionaire. At this occasion the fieldworkers take the GPS coordinates of the study participant's homes and nearest spraying areas. The three follow-up urine samples between baseline and follow-up and the exposure activity questionnaires will then be administered.

The farm-owner questionnaire is administered three times during the study. The first interview was conducted in November 2017 to characterize the farms according to their production system in place and ask participants to provide a copy of their spraying records for 2016/2017 and a copy of their spraying calendars for $2017 / 2018$. Subsequently, the farms are visited and asked 
to provide a copy of the spraying records in June 2018 and June 2019.

The air and water sampling are conducted during the first phase of the baseline study between July 2017 and June 2018.

\section{Data analysis}

\section{Sample size calculations}

Neurobehavioural outcomes The sample size for neurobehavioral health outcomes is determined assuming a 0.2 standard deviation from the median neurobehaviour score in the exposed group compared to the control group. This corresponds to observed differences found in studies investigating environmental exposure on neurobehavioral outcomes, using metabolites as the method to assess for pesticide exposure in the Western Cape of South Africa [45-47] in the United States [48] and in Costa Rica $[49,50]$. A sample size of 900 was judged to be adequate with a power of $80 \%$ and a $5 \%$ level of significance.

Reproductive hormone outcomes Sample size calculations for reproductive outcomes were determined using findings from a previous cross-sectional study conducted in the same study areas [16] that showed differences in the same reproductive outcomes as in this study between farm and non-farm residing boys. A two sample-test of equality of means is used (exposed: control ratio $=2: 1$, i.e. to ensure that more participants are recruited from pesticide exposed areas, power $=80 \%$, confidence level $=95 \%$ ). The reproductive outcome requiring the highest sample size to show a significant difference in farm versus non-farm boys is for serum testosterone (one of the five hormones to be measured) for which a sample of 498 (i.e. 332 exposed and 166 unexposed) participants are required to ensures sufficient power for the boys in the study. The sample size calculations for boys were considered applicable for girls as all the clinical outcomes were similar and therefore a sample size of 500 girls was targeted to ensure adequate power to test associations between exposures and outcomes.

\section{Data monitoring}

The data monitoring is independent of sponsors. The field coordinator and fieldworkers upload the surveys to the server, while the $\mathrm{PhD}$ and post-doctoral students together with the principal investigators monitor the data when uploaded.

\section{Statistical analysis}

Associations between pesticide exposure levels and the obtained health outcome will be conducted by considering relevant confounders. Outcomes of interest include reproduction and pubertal growth (levels of reproductive hormones; sexual maturity rating, height, weight, BMI). The neurobehavioural primary endpoint is cognitive functioning with two secondary endpoints, psychosocial and emotive functioning, health and well-being. Pesticide exposures of interest include bio-monitoring measurements, and the pesticide exposure indices derived from pesticide related risk factors including proximity to the field, contact with the field, involvement in farming activities and contact with the parent or guardian. Co-exposures of interest include e-media use including owning a smart phone and or electronic media device/s, internet use and specific involvement in internet activities such as online games. Another co-exposure of interest includes alcohol consumption during pregnancy characterised by maternal prenatal, perinatal and postnatal alcohol use. Relevant confounders of interest include medical history and current health status, diet, developmental history and indoor chemical use/pollution.

Pesticide exposure will be characterised and compared according to following five different levels: (i) self-reported exposure obtained with the participant questionnaire (e.g., reported behavioral exposure profiles); (ii) self-reported exposure obtained with the guardian questionnaire (e.g., farm worker versus non-farm workers; living on a farm; GPS coordinates of the household and proximity to agricultural fields); (iii) concentration of metabolites and active ingredients measured in urine and hair samples of children; (vi) collected spraying plans and records from farm-owner interviews (to establish pesticide emission profiles for apple, table grapes, wheat and citrus farms and develop a Crop Exposure Matrix (CEM); and (v) measured concentration of active ingredients in passive air and water samples.

Firstly, cross-sectional analyses at baseline and final-follow-up will be conducted. Methods including multiple imputations will be used to address any missing data in the analysis. Further, various types of longitudinal analyses will be conducted. Change analyses will consider whether changes in exposures are related in changes in outcomes. A cohort approach is applied to explore whether exposure at baseline results in new incident cases and provides us the opportunity to assess developmental processes during the time of follow-up. Either clinical case definition is used or in the absence of the criteria for a specific outcome, a priori defined cut-off is used such as the 75th percentile.

Depending on the outcome, logistic, linear or ordinal regression modeling will be conducted.

To maximize power outcomes and exposure, a regression model on a continuous scale (linear/ordinal), will be considered whenever possible. The form of the exposure-response relationships will be explored using polynomial terms or non-parametric approaches (splines). In supplementary analyses, outcomes will be 
dichotomised and logistic regression modeling will be used. Exposure variables may be dichotomised or categorised for easier communication if suitable.

\section{Study population}

In total, 1400 invite letters were returned from the parents who showed interest to participate. 1001 study participants and their guardian/parents from this pool consented and took part in the baseline examination between April and September 2017. Table 2 gives an overview about the study population and basic demographic information.

Table 2 provides descriptive data on the participants in this study, showing they are close to equally distributed amongst the three study areas. The participants age ranges from 9 to 16 years with the highest numbers, almost $60 \%$ falling in the younger category of 9-11 years. Their gender is almost equally distributed, with $5 \%$ more females than males. The grades range from 2nd to 9th grade, with $66 \%$ of the participants falling within the middle category, 4th-6th grade. $46 \%$ of the participants live on a farm and $66 \%$ have a family member who is a farm worker. Further regarding pesticide exposure, 80\% of the participants have said yes to having ever seen pesticide spraying activities in the nearby field; $23 \%$ have responded to having helped with cleaning farm equipment in the past; and $20 \%$ of the participants have assisted with pesticide storage in the past 7 days. In terms of the participants engagemement with electronic media use, specifically mobile phones, $31 \%$ use a phone and of those who use a phone, $89 \%$ use smart phones. The majority of these users live in the 1st study area, Grabouw, and are the majority who engage with activities on their phones including watching videos, playing online games and listening to music.

\section{Discussion}

The main strength of this study is its longitudinal prospective design providing the possibility to determine the varying effect of the pesticide exposure, media use and other exposures over different time points. This study also collected detailed objective exposure data on current used agricultural pesticides obtained from urine and hair bio-monitoring, as well as environmental passive air and water sampling in the study areas. This exposure data will enable us to characterise and quantify the level of exposure of the participants and assess their cumulative exposure over the follow-up period.

By combining the biomonitoring with spraying schedules and detailed questionnaire data, a better understanding of critical behaviours for pesticide exposure will be obtained. All study areas are economically important farming areas with intensive use of pesticides, where pesticides have been detected previously and from the results we have attained an equal distribution of participants across these areas. By selecting three different areas where several types of farming products are cultivated, the study offers the possibility to compare the effects of different types and mixtures of pesticides. The study includes almost half of participants from farms compared to children not living on a farm, yielding variations in levels of exposure amongst the cohort. Useful exposure contrasts within the cohort is also demonstrated in other items of the baseline survey, such as seeing spraying activities and engaging with pesticide equipment which was reported by a few participants. About a third of the participants do engage with media use in all three areas, even though these are low income rural areas. Thus, the cohort is well suited to study the effects of uptake of media in adolescence.

The study population has an appropriate age range that includes children in various stages of development which will enable the researchers to investigate the changes in periods of pubertal and neurobehavioral development amongst the distinct groups of exposed children over the two-year period.

Development stage is assessed using standardised methods and complemented with a wide range of hormonal measurements indicative of reproductive development obtained from blood samples. Use of a validated computerised tool to measure neurobehaviour is a further asset of this study. This is the first iPad based study on this topic in a rural setting which will yield evidence of standardizing quality data and reliability for future studies on a large scale. Additionally, the questionnaires are comprehensive for collecting data on children's diet, socio-economic status, prenatal exposures and family environment to determine any influencing factors on behavior and development. The generation of exposure indices and the area of residence will help in understanding the patterns of lifetime exposure in relation to different environmental factors for e.g. proximity to spraying area.

Furthermore, by including co-exposures, pesticide effects can be studied independent of e-media and alcohol consumption, while synergistic effects can be studied.

Lastly this study carries power for attaining its objectives and methods with the use of a 1000 sample population.

Results from this study will be used to educate the community and government sectors involved in pesticide use and regulation. Suggestions that arise from this study will provide farming communities with awareness of health promotion and prevention strategies. In conclusion the findings from this study can contribute to the improvement and protection of children's health and development locally and internationally as these pesticides are used globally. This will be the first longitudinal study investigating the reproductive health effects on children of agricultural pesticides in current use and will seek to address conflicting results from studies investigating neurobehavioural effects. 


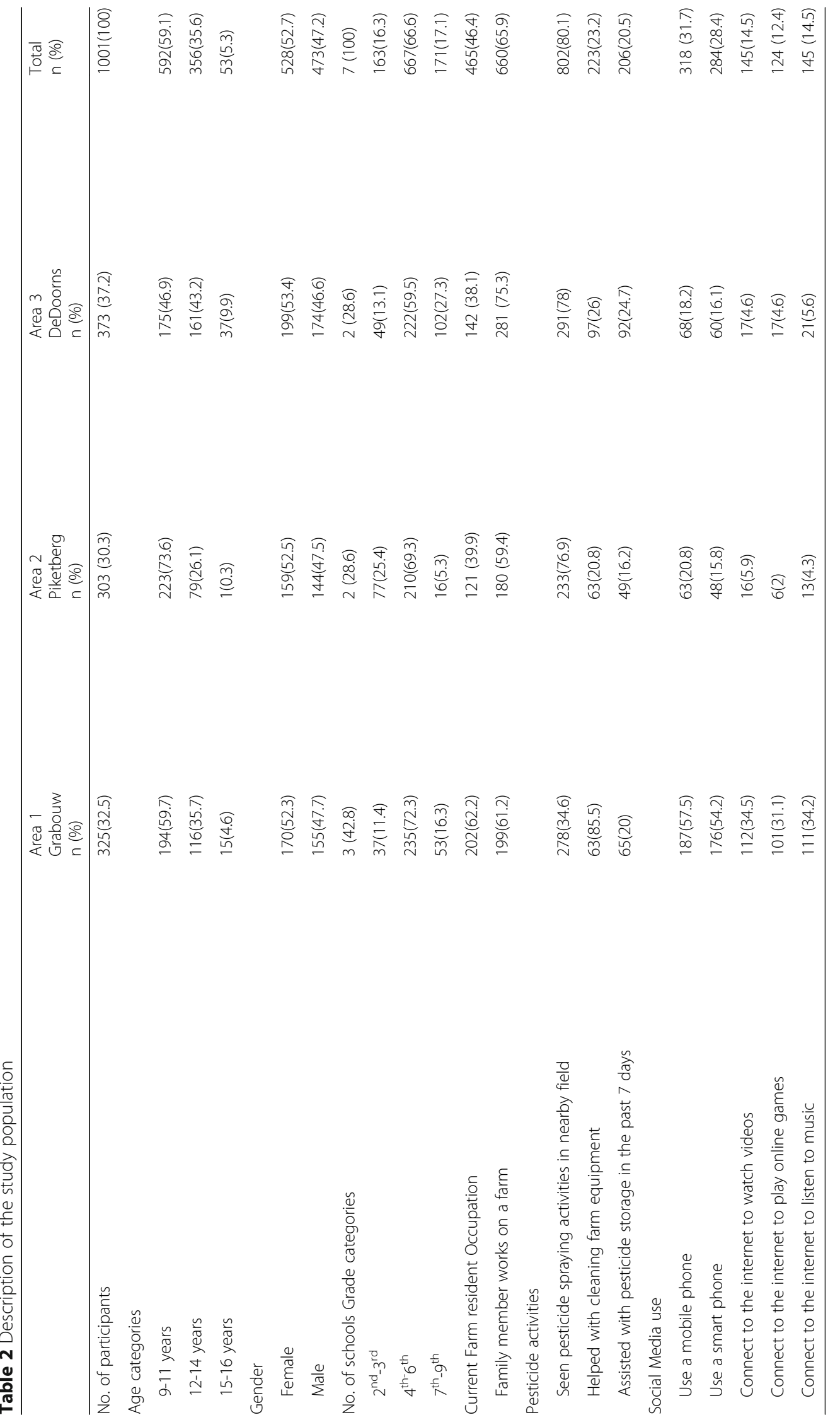




\section{Abbreviations}

ADHD: Attention Deficit Hyperactivity Disorder; ASD: Autism Spectrum Disorder; CANTAB: CAmbridge Neuropsychological Test Automanted Battery; CEM: Crop-exposure matrices; DAFF: Department of Agriculture, Forestry and Fisheries; EDC: Endocrine Disrupting Chemical; FASD: Fetal Alcohol Syndrome Disorder; HIT-6: Headache Impact Test-6; ODK: Open Data Kit; PDD: Pervasive Developmental Disorders; PUF-PAS: Polyurethane foam disks; SDB: Styrenedivinylbenzene; WHO: World Health Organisation; XAD-PAS: XAD resin

\section{Acknowledgements}

We acknowledge the research chair principal investigators and funding sources in support of this study. We thank all experts who have contributed to the body of knowledge for this study. We are grateful to all councils for ethical guidance and approval of the study and the agricultural organisations who have supported the implementation of this study in the field. Thanks to all the school governing bodies for the grace extended to our study needs and to all the participants who have willingly offered valuable contribution to make this study a success. Lastly, we acknowledge and extend gratitude toward the fieldworkers, Lindile Masinyana, Muneebah Dawson, Philancia Januari and field coordinators, Wisdom Basera and Samuel Fuhrimann, in this study who have persevered and made the data collection a success for the outcomes of this study.

\section{Funding}

This project is imbedded within the South African-Swiss Bilateral SARChi in Global Enviromental Health of Professor Aqiel Dalvie (PhD), Centre for Environmental and Occupational Health Research, University of Cape Town and Professor Martin Röösli (PhD), Swiss Tropical and Public Health Institute. This chair was formed in 2015 with funding sources from SA National Research Foundation (NRF), SARChi Chair Programme, Swiss State Secretariat for Education, Research and Innovation, University of Basel and the Swiss TPH. Additional funding includes the Swiss Government Federation, ESKAS, the South African Medical Research Foundation Self-Initiated Research Programme, SA NRF Competitive Programme for Rated Researchers, the Swiss - African Research Cooperation (SARECO), NRF Incentive Programme for Rated Researchers and the SA Department of Science and Technology.

\section{Availability of data and materials}

The datasets generated and/or analysed during the current study are not publicly available due to confidentiality and anonymity of the learners and their guardians. The datasets are stored on a secure Alfresco website and are available from the authors and to the co-authors on reasonable request.

\section{Authors' contributions}

$A D$ and $M R$ are the principal investigators of the study and contributed to the design, methods, funding and topics in the protocol and writing of the manuscript. SM is responsible for writing up the first draft and editing of the protocol paper during its development toward the final version, baseline data monitoring, the descriptive analysis, establishing the methods and training for the cognitive assessment and development of the questionnaires. WB and SF developed the SOPs and coordinated the cohort study. SF was responsible for the methods of the water and air sampling and the farm-owner questionnaire. WB developed the reproductive and pesticide bio-monitoring and exposure questionnaire aspects of the proposal and was responsible for the methods of the reproductive health outcome. MM and JVW and SD are responsible for the laboratory analysis of hair, urine and blood samples for the study. They contributed to the section on pesticide biomonitoring and sample testing in this paper. NP and the above authors read and provided comments for the protocol draft. All authors have read and approved the final version of the protocol paper.

\section{Ethics approval and consent to participate}

Parents or guardians of the participants as well as farm-owner were required to provide signed permission to be a part of this study and written consent. The participant learner were required to provide written assent once their parent's consent was received and before any data was collected. Primary ethical principles based on the Helskinki Declaration [51], will be considered for the benefit and protection of subjects in this study.

The ethical protocols for the study on pesticide exposure and reproductive health outcomes has been approved by the University of Cape Town's
Human Research Ethics Committee (HREC reference number: 234/2009). An amendment was made to this protocol for the addition of neurobehavioral outcome and submitted for ethical clearance, which was approved on May 2017 by the University of Cape Town's Human Research Ethics Committee (reference: 234/2009). The Ethics Committee of the Northwest and Central Switzerland (EKNS) reviewed and confirmed ethical and scientific standards of the study in November 2017 (reference: EKNS 2017-01683). The Western Cape Education Department has provided approval and consent to conduct this study amongst the children who attend school in these study areas (reference: 20150629-846). An additional ethical protocol for the farm-owner questionnaire and the environmental assessment was approved by HREC on the October 2017 (reference number: 597/2017).

\section{Consent for publication}

"Not applicable"

\section{Competing interests}

The authors declare that they have no competing interests in this study.

\section{Publisher's Note}

Springer Nature remains neutral with regard to jurisdictional claims in published maps and institutional affiliations.

\section{Author details}

${ }^{1}$ Centre for Environment and Occupational Health Research, School of Public Health and Family Medicine, Faculty of Health Sciences, University of Cape Town, Anzio Road, Cape Town, South Africa. ${ }^{2}$ Swiss Tropical Public Health Institute, Basel, Switzerland. ${ }^{3}$ University of Basel, Basel, Switzerland. ${ }^{4}$ Department of Paediatrics and Child Health, University of Cape Town, Cape Town, South Africa. ${ }^{5}$ Hair and Skin Research Laboratory, University of Cape Town and Groote Schuur Hospital, Cape Town, South Africa.

Received: 27 March 2018 Accepted: 29 June 2018

Published online: 11 July 2018

\section{References}

1. Eddleston M, Karalliedde L, Buckley N, Fernando R, Hutchinson G, Isbister G, et al. Pesticide poisoning in the developing world-a minimum pesticides list. Lancet Lond Engl. 2002;360:1163-7.

2. Owens K, Feldman J, Kepner J. Wide range of diseases linked to pesticides. Pestic You. 2010;30:13-21.

3. Bjørling-Poulsen M, Andersen HR, Grandjean P. Potential developmental neurotoxicity of pesticides used in Europe. Environ Health. 2008:7:50. https://doi.org/10.1186/1476-069X-7-50.

4. Quinn LP, Van Den Berg J, Fernandes-Whaley M, Roos C, Bouwman H, Kylin $\mathrm{H}$, et al. Pesticide use in South Africa: one of the largest importers of pesticides in Africa. In: Pesticides in the Modern World-Pesticides Use and Management 2011: InTech. https://doi.org/10.5772/16995.

5. Dalvie M. Reproductive health effects of contemporary pesticides used in South Africa. Res J Chem Environ. 2014;18:77-82.

6. WHO Endocrine Disrupting Report 2012.pdf n.d.

7. WHO. Children'S health and the Environment 2008.

8. Rother H-A, Hall R, London L. Pesticide use among emerging farmers in South Africa: contributing factors and stakeholder perspectives. Dev South Afr. 2008;25(4):399-424. https://doi.org/10.1080/03768350802318464.

9. Ecobichon DJ. Pesticide use in developing countries. Toxicology. 2001;160: 27-33. https://doi.org/10.1016/50300-483X(00)00452-2.

10. DAFF. Pesticide Management Policy for South Africa. South Africa; 2010

11. Dabrowski J. Development of pesticide use maps for SA. South Afr J Sci. 2015;111:7.

12. Dalvie MA, Cairncross E, Solomon A, London L. Contamination of rural surface and ground water by endosulfan in farming areas of the western cape, South Africa. Environ Health. 2003;2(1):1. https://doi.org/10.1186/1476-069X-2-1.

13. Dalvie MA, Naik I, Channa K, London L. Urinary dialkyl phosphate levels before and after first season chlorpyrifos spraying amongst farm workers in the Western Cape, South Africa. J Environ Sci Health Part B. 2011;46(2):163-72.

14. Dalvie MA, Sosan MB, Africa A, Cairncross E, London L. Environmental monitoring of pesticide residues from farms at a neighbouring primary and preschool in the western cape in South Africa. Sci Total Environ. 2014:466-467: 1078-84. https://doi.org/10.1016/.jscitotenv.2013.07.099. 
15. Dalvie MA, Africa A, Solomons A, London L, Brouwer D, Kromhout $H$. Pesticide exposure and blood endosulfan levels after first season spray amongst farm workers in the western cape, South Africa. J Environ Sci Health Part B. 2009:44:271-7. https://doi.org/10.1080/03601230902728351.

16. English RG, Perry M, Lee MM, Hoffman E, Delport S, Dalvie MA. Farm residence and reproductive health among boys in rural South Africa. Environ Int. 2012;47:73-9. https://doi.org/10.1016/j.envint.2012.06.006.

17. Heeren GA, Tyler J, Mandeya A. Agricultural chemical exposures and birth defects in the eastern Cape Province, South Africa a case - control study. Environ Health. 2003;2:11. https://doi.org/10.1186/1476-069X-2-11.

18. Roser K, Schoeni A, Foerster M, Röösli M. Problematic mobile phone use of Swiss adolescents: is it linked with mental health or behaviour? Int J Public Health. 2016;61:307-15. https://doi.org/10.1007/s00038-015-0751-2.

19. Schoeni A, Roser K, Röösli M. Symptoms and cognitive functions in adolescents in relation to mobile phone use during night. PLoS One. 2015; 10:e0133528. https://doi.org/10.1371/journal.pone.0133528.

20. Zheng F, Gao P, He M, Li M, Wang C, Zeng Q, et al. Association between mobile phone use and inattention in 7102 Chinese adolescents: a population-based cross-sectional study. BMC Public Health. 2014;14:1022. https://doi.org/10.1186/1471-2458-14-1022.

21. Schoeni A, Roser K, Röösli M. Symptoms and the use of wireless communication devices: a prospective cohort study in Swiss adolescents. Env Res. 2017;154:275-83.

22. UNDP. Human Development Report 2015. 2015.

23. May PA, Gossage JP, Brooke LE, Snell CL, Marais A-S, Hendricks LS, et al. Maternal risk factors for fetal alcohol syndrome in the western Cape Province of South Africa: a population-based study. Am J Public Health. 2005;95:1190-9. https://doi.org/10.2105/AJPH.2003.037093.

24. London L. The "dop" system, alcohol abuse and social control amongst farm workers in South Africa: a public health challenge. Soc Sci Med. 1982; 1999(48):1407-14.

25. Gossage J, Snell C, Parry C, Marais A-S, Barnard R, de Vries M, et al. Alcohol use, working conditions, job benefits, and the legacy of the "Dop" system among farm Workers in the Western Cape Province, South Africa: hope despite high levels of risky drinking. Int J Environ Res Public Health. 2014;11: 7406-24. https://doi.org/10.3390/ijerph110707406.

26. Robbins TW, James M, Owen AM, Sahakian BJ, Mclnnes L, Rabbitt P. Cambridge neuropsychological test automated battery (CANTAB): a factor analytic study of a large sample of normal elderly volunteers. Dement Basel Switz. 1994;5:266-81.

27. Elliott R, Sahakian BJ, Matthews K, Bannerjea A, Rimmer J, Robbins TW. Effects of methylphenidate on spatial working memory and planning in healthy young adults. Psychopharmacology. 1997;131:196-206.

28. Roberts AC, De Salvia MA, Wilkinson LS, Collins P, Muir JL, Everitt BJ, et al. 6Hydroxydopamine lesions of the prefrontal cortex in monkeys enhance performance on an analog of the Wisconsin card sort test: possible interactions with subcortical dopamine. J Neurosci. 1994;14:2531-44.

29. Jamal GA, Hansen S, Pilkington A, Buchanan D, Gillham RA, Abdel-Azis M, et al. A clinical neurological, neurophysiological, and neuropsychological study of sheep farmers and dippers exposed to organophosphate pesticides. Occup Environ Med. 2002:59:434-41. https://doi.org/10.1136/oem.59.7.434.

30. Goldberg MC, Mostofsky SH, Cutting LE, Mahone EM, Astor BC, Denckla $M B$, et al. Subtle executive impairment in children with autism and children with ADHD. J Autism Dev Disord. 2005;35:279-93. https://doi. org/10.1007/s10803-005-3291-4.

31. Green CR, Mihic AM, Nikkel SM, Stade BC, Rasmussen C, Munoz DP, et al Executive function deficits in children with fetal alcohol spectrum disorders (FASD) measured using the Cambridge neuropsychological tests automated battery (CANTAB). J Child Psychol Psychiatry. 2009;50:688-97. https://doi. org/10.1111/j.1469-7610.2008.01990.x

32. Rohlman DS, Gimenes LS, Eckerman DA, Kang S-K, Farahat FM, Kent Anger W. Development of the behavioral assessment and research system (BARS) to detect and characterize neurotoxicity in humans. NeuroToxicology. 2003; 24:523-31. https://doi.org/10.1016/S0161-813X(03)00023-8.

33. Ravens-Sieberer U, Erhart M, Rajmil L, Herdman M, Auquier P, Bruil J, et al. Reliability, construct and criterion validity of the KIDSCREEN-10 score: a short measure for children and adolescents' well-being and health-related quality of life. Qual Life Res. 2010;19:1487. https:/doi.org/10.1007/s11136-010-9706-5.

34. Foerster M, Roser K, Schoeni A, Röösli M. Problematic mobile phone use in adolescents: derivation of a short scale MPPUS-10. Int J Public Health. 2015; 60:277-86. https://doi.org/10.1007/s00038-015-0660-4.
35. Durusoy R, Hassoy H, Özkurt A, Karababa AO. Mobile phone use, school electromagnetic field levels and related symptoms: a cross-sectional survey among 2150 high school students in Izmir. Environ Health Glob Access Sci Source. 2017:16:51. https://doi.org/10.1186/s12940-017-0257-x.

36. Stalin P, Abraham SB, Kanimozhy K, Prasad RV, Singh Z, Purty AJ. Mobile phone usage and its health effects among adults in a semi-urban area of southern India. J Clin Diagn Res JCDR. 2016;10:LC14-6. https://doi.org/10. 7860/JCDR/2016/16576.7074

37. Mohammadianinejad SE, Babaei M, Nazari P. The effects of exposure to low frequency electromagnetic fields in the treatment of migraine headache: a cohort study. Electron Physician. 2016;8:3445-9. https:/doi.org/10.19082/3445.

38. Goodman A, Lamping DL, Ploubidis GB. When to use broader internalising and externalising subscales instead of the hypothesised five subscales on the strengths and difficulties questionnaire (SDQ): data from British parents, teachers and children. J Abnorm Child Psychol. 2010;38:1179-91. https://doi. org/10.1007/s10802-010-9434-x

39. Marshall WA, Tanner JM. Variations in pattern of pubertal changes in girls. Arch Dis Child. 1969;44:291-303.

40. Graham SD, Keane TE, Glenn JF. Glenn's urologic surgery. Philadelphia: Lippincott Williams \& Wilkins; 2010.

41. Baldi I, Carles C, Blanc-Lapierre A, Fabbro-Peray P, Druet-Cabanac M, Boutet-

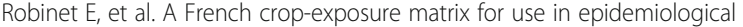
studies on pesticides: PESTIMAT. J Expo Sci Environ Epidemiol. 2017;27:5663. https://doi.org/10.1038/jes.2015.72.

42. Hayward SJ, Gouin T, Wania F. Comparison of four active and passive sampling techniques for pesticides in air. Environ Sci Technol. 2010:44:34106. https://doi.org/10.1021/es902512h.

43. Moschet C, Vermeirssen ELM, Singer H, Stamm C, Hollender J. Evaluation of in-situ calibration of Chemcatcher passive samplers for 322 micropollutants in agricultural and urban affected rivers. Water Res. 2015;71:306-17. https:// doi.org/10.1016/j.watres.2014.12.043.

44. Ochieng AA, Dalvie MA, Little F, Kromhout H. Relationship between environmental exposure to pesticides and anthropometric outcomes of boys in the rural western cape, South Africa. S Afr Med J. 2013;103:942-7.

45. Holtman Z. Neurobehavioural effects of pesticide exposure among emerging farmers in the western cape 2013.

46. London L, Beseler C, Bouchard MF, Bellinger DC, Colosio C, Grandjean P, et al. Neurobehavioral and neurodevelopmental effects of pesticide exposures. Neurotoxicology. 2012;33:887-96. https://doi.org/10.1016/j.neuro.2012.01. 004.

47. Motsoeneng PM, Dalvie MA. Relationship between urinary pesticide residue levels and neurotoxic symptoms among women on farms in the western cape, South Africa. Int J Environ Res Public Health. 2015;12:6281. https://doi. org/10.3390/ijerph120606281.

48. Grandjean P, Landrigan PJ. Neurobehavioural effects of developmental toxicity. Lancet Neurol. 2014;13:330-8. https://doi.org/10.1016/S14744422(13)70278-3.

49. Fuhrimann S, Winkler M, Staudacher P, Weiss FT, Stamm C, EggenR RIL, et al. Exposure to pesticides and health effects in farm owners and workers from conventional and organic agricultural farms in Costa Rica: a study protocol. JMIR Research Protocols. Toronto: JMIR Publications Inc; 2018. https://doi. org/10.2196/preprints.10914.

50. van Wendel de Joode B, Mora AM, Lindh CH, Hernández-Bonilla D, Córdoba L, Wesseling C, et al. Pesticide exposure and neurodevelopment in children aged 6-9 years from Talamanca, Costa Rica. Cortex. 2016;85:137-50. https://doi.org/10.1016/j.cortex.2016.09.003.

51. World Medical Association Declaration of Helsinki. Ethical principles for medical research involving human subjects. JAMA. 2013;310:2191-4. https://doi.org/10.1001/jama.2013.281053. 